\title{
Métodos de excavación: del trabajo de campo a la interpretación arqueológica
}

\author{
Rafael Mora Torcal, Jorge Martínez Moreno, Xavier Roda Gilabert, \\ Miquel Roy Sunyer, Susana Vega Bolívar ${ }^{1}$
}

Recibido: 18-10-2014

Aceptado: 10-11-2014

\section{Resumen}

El registro arqueológico es la base material sobre la que se interpreta el pasado. Su recuperación ha sido foco de discusión metodológica y en los últimos años se ha visto impulsado por la irrupción de las nuevas tecnologías. En este trabajo exponemos la metodología de campo seguida por el Centre d'Estudis del Patrimoni Arqueològic de la Prehistòria (CEPAP-UAB). La excavación en extensión se presenta como propuesta metodológica para la recuperación del registro arqueológico. A continuación se expone la sistemática para la adquisición, digitalización, integración de los datos y la posterior generación de mapas temáticos dirigida a interpretar la relación entre los distintos ítems o estructuras. La metodología mostrada sirve como base para la creación de hipótesis de trabajo que se contrastan a lo largo del trabajo de campo y sustentan la base de la investigación.

Palabras clave: métodos de registro; aplicaciones informáticas; arqueografía; yacimientos paleolíticos.

Resum. Mètodes d'excavació: del treball de camp a la interpretació arqueològica

El registre arqueològic es la base material a partir de la qual s'interpreta el passat. La seva recuperació ha estat el focus de discussió metodològica i en els darrers anys s'ha vist impulsat per la irrupció de les noves tecnologies. En aquest treball exposem la metodologia de camp seguida pel Centre d'Estudis del Patrimoni Arqueològic de la Prehistòria (CEPAP-UAB). L'excavació en extensió es presenta com a proposta metodològica per a la recuperació del registre arqueològic. A continuació s'exposa la sistemàtica per a l'adquisició, digitalització, integració de les dades i generació posterior de mapes temàtics dirigida a interpretar la relació entre els diferents ítems o estructures. La metodologia presentada és la base per a la creació d'hipòtesis de treball que es contrasten al llarg del treball de camp i sustenten la base de la investigació.

Paraules clau: mètodes de registre; aplicacions informàtiques; arqueografia; jaciments paleolítics.

1. Centre d'Estudis del Patrimoni Arqueològic de la Prehistòria (CEPAP-UAB). Universitat Autònoma de Barcelona. 08193 Bellaterra (Cerdanyola del Vallès).

rafael.mora@uab.cat; jorge.martinez@uab.cat; javier.roda@uab.cat; miquel.roy@uab.cat; susana.vega@uab.cat 
Abstract. Excavation methods: from fieldwork to the archaeological interpretation

The archaeological record is the material basis on which the past is interpreted. His recovery has been the focus of methodological discussion and in recent years has been driven by the emergence of new technologies. In this paper we present the fieldwork methodology followed by the Centre d'Estudis del Patrimoni Arqueologic de la Prehistòria (CEPAP-UAB). The surface excavation is presented as a methodological proposal for the recovery of the archaeological record. Below is exposed to systematic for the acquisition; digitization; data integration and the subsequent generation of thematic maps that aimed to interpret the relationship between the different items or structures. The methodology shown constitutes the basis for creating working hypotheses are contrasted throughout the fieldwork and support the research base.

Keywords: Fieldwork recording systems; Computer applications; Archaeography; Palaeolithic sites.

Mora Torcal, Rafael; Martínez Moreno, Jorge; Roda Gilabert, Xavier; Roy Sunyer, Miquel; Vega Bolívar, Susana. «Métodos de excavación: del trabajo de campo a la interpretación arqueológica». Treballs d’Arqueologia, 2014, núm. 20, p. 7-20. DOI: 10.5565/rev/tda.53

\section{Introducción}

El registro arqueológico constituye la base a partir de la cual se interpretan las sociedades del pasado, pero es un elemento único y finito. Es por este motivo que su recuperación ha sido y sigue siendo objeto de discusión y renovación teórica constante. La irrupción de nuevas tecnologías está conllevando profundas modificaciones en todas las disciplinas y no es ajeno a ello el proceso de recuperación y sistematización de los datos arqueológicos.

Tradicionalmente, se ha considerado la excavación arqueológica y todos los procesos asociados a la obtención y gestión de la información como algo propio de cada investigador, o de cada yacimiento. Esto ha generado una enorme diversidad de planteamientos y comportamientos en nuestra profesión, que van unidos a la propia evolución histórica de la arqueología. En este sentido, no es posible disociar yacimientos arqueológi- cos, época histórica en la que se llevaron a cabo las excavaciones, personalidad y formación del investigador que realizó los trabajos e información contextual de la que se dispone actualmente. A pesar de los esfuerzos realizados por parte de los profesionales de la arqueología para asumir unos planteamientos comunes mínimos a partir de los cuales desarrollar la investigación arqueológica, nos encontramos con problemas fundamentales que impiden homogeneizar estos criterios.

En nuestro país, el rol de las universidades en la creación, difusión y homogeneización de los métodos y las técnicas básicas en la recuperación del registro arqueológico ha sido nulo hasta hace pocos ańos. La puesta en marcha de plan Bolonia y el equiparamiento con Europa han conllevado tímidamente el inicio de una formación en el ámbito de las metodologías y técnicas del trabajo de campo. Sin embargo, sigue ocupando un lugar 
secundario dentro de las enseñanzas académicas y se deja que sea el propio sujeto el que, a través de su iniciativa, genere su experiencia de campo. El resultado es una enorme disparidad de criterios y poca renovación de los parámetros constructivos en esta área.

Si analizamos el papel que desempeñan hoy día las excavaciones de urgencia, que son el principal foco de recuperación del registro arqueológico, nos daremos cuenta de que no existen los planteamientos teóricos y metodológicos básicos para abordar este trabajo. Los factores negativos que inciden en este problema son, por un lado, la escasa formación de los equipos, y, por el otro, la escasez de infraestructuras básicas debido a la ausencia de soporte institucional. A esto hay que ańadir el papel que tienen las empresas en la mercantilización del patrimonio cultural y la presión económica que ejercen sobre el colectivo arqueológico.

Por todo esto, proponemos que se ha de reflexionar, en primer lugar, sobre las distintas estrategias desarrolladas para la sistematización de la recuperación del registro arqueológico; en segundo lugar, sobre el papel que deben desempeñar las distintas partes implicadas, las universidades (formación e investigación); las empresas (papel en la recuperación y estrategias en las que se enmarcan); la administración (como ente que vela por la correcta gestión del patrimonio); y, finalmente, los profesionales (como motores básicos de la investigación y principales implicados en el proceso de excavación, estudio y divulgación del registro arqueológico). Este planteamiento servirá para mejorar las relaciones entre la investigación y la sociedad, porque permitirá establecer unos criterios homogéneos y crear unos estándares de trabajo. Por tanto, también facilitará la tarea de las empresas de arqueología que, en la actual coyuntura, son las principales encargadas de la recuperación del registro arqueológico.

Después de una larga tradición histórica de disparidad de criterios en lo que respecta a la metodología más básica de la disciplina, consideramos que es fundamental reunir a debate las distintas partes implicadas en este proceso. La recuperación, estudio y gestión del registro arqueológico compete a distintas instituciones con objetivos y protocolos diversos, por lo que es urgente establecer criterios estándar de actuación. Aunque se han realizado reuniones, seminarios y congresos sobre la recuperación del registro arqueológico, en su mayor parte se limitan a la simple presentación de novedades técnicas. Este artículo pretende superar esta visión excesivamente tecnológica y propone como objetivo la reflexión sobre el significado del registro arqueológico como sustento para establecer una base teórica, metodológica y técnica común a todas las partes implicadas $y$, en consecuencia, favorecer la sinergia entre ellas.

\section{Del cuaderno de campo al registro digital}

A lo largo de los últimos 25 años, asistimos a profundos cambios metodológicos y técnicos que han afectado especialmente a las actividades relacionadas con el trabajo de campo y la recuperación del registro arqueológico. Inicialmente, la creación y gestión de bases de datos implicaban el uso de lenguajes de programación Fortran o Basic, así como el uso de tarjetas perforadas para cada obje- 
to arqueológico, y su posterior procesado en los centros de cálculo.

La aplicación de las nuevas tecnologías en la sistemática de recuperación del registro arqueológico, así como el desarrollo de la microinformática, han revolucionado completamente el procedimiento. Los actuales equipos no solo son capaces de procesar y gestionar este tipo de bases de datos, sino que además conllevan enormes ventajas a la hora de abordar la cuestión de la recogida de la información arqueológica.

Desde nuestra experiencia y capacidad hemos seguido un proceso de complejidad creciente en las técnicas de recuperación y registro de materiales arqueológicos. Durante ańos nos vimos obligados a desarrollar entornos estadísticos y gráficos para gestionar los datos arqueológicos. Actualmente apostamos por aplicar determinados entornos de gestión y graficado informático desarrollados en el mercado, y que no necesariamente están relacionados con la arqueología, pero cuyas características permiten una fácil aplicación.

En el ámbito metodológico, nuestra perspectiva pasa por poner de relieve el significado de los ítems arqueológicos a partir de su contextualización. Esto implica considerar la recuperación del registro arqueológico como una parte relevante dentro del proceso de investigación y que está estrechamente relacionada con su interpretación. Esta noción de recuperación contextual facilita que esa información pueda ser reinterpretada en el futuro.

La sistemática que proponemos se ha centrado de forma prioritaria en la recogida de información de asentamientos del plio-pleistoceno, pleistoceno y holoceno, especialmente en sociedades cazadorasrecolectoras. Para ello, hemos desarro- llado un protocolo de trabajo con unos parámetros uniformes y coordinados por los principios de la excavación en extensión, en los que la información contextual (especialmente de orden geológico) es esencial a la hora de analizar la dimensión tridimensional de los ítems arqueológicos (ya sean artefactos o ecofactos).

Paralelamente, queremos insistir en la necesidad de homogeneizar los sistemas de recogida de información y su posterior procesado. Aunque se han hecho intentos de sistematización - muchos de los cuales comparten una filosofía similar en el tratamiento de los datos arqueológicos_-, no se ha consensuado un sistema unificado. A nuestro entender, la ausencia de una metodología común impide el intercambio de información entre proyectos y limita la posibilidad de comparar ámbitos y registro.

\section{La posición de los objetos}

Cuando empezamos a trabajar en prehistoria, el uso de las coordenadas cartesianas en la recuperación de los ítems era una novedad técnica. Este sistema implica el levantamiento individual de artefactos o estructuras en base a uno o varios puntos tridimensionales, siendo necesario un nivel óptico y montar una cuadrícula terrestre o aérea (Méroc et al., 1954; Laplace, 1971).

La cuadrícula, aérea o terrestre, se posicionaba en el plano horizontal dividiendo la superficie a excavar en áreas de $1 \mathrm{~m}^{2}$ que se identificaban a partir de la combinación de letras y números a fin de identificar sus ejes. Para tomar la profundidad, se definía un punto arbitrario - 0 - a partir del cual se referenciaban todas las cotas. Los objetos se posiciona- 
ban mediante coordenadas cartesianas relativas al ángulo inferior izquierdo de cada metro cuadrado; o se posicionaban globalmente usando tallas. Cada talla incluía los artefactos encontrados entre los valores máximo (base) y mínimo (techo) de la variable profundidad con respecto a la cota $-0-$, con una amplitud que habitualmente se situaba en los $10 \mathrm{~cm}$. El objetivo en la excavación era llegar en todos los cuadros de la superficie excavada a una determinada cota.

Esta estrategia supone un problema implícito en la propia concepción de la aplicación del sistema de tallas, dado que estas se conformaban en una delineación horizontal sin tener en cuenta que las unidades arqueológicas raramente presentan tal geometría, por lo que no era inusual la mezcla entre distintos niveles. Por otro lado, la excavación con este método es rápida, transfiriendo gran parte del trabajo al proceso de siglado e inventariado de los objetos arqueológicos.

Con esta estrategia era difícil llevar a cabo planimetrías, siendo necesario transformar las variables alfabéticas en numéricas y las coordenadas relativas de cada ítem en valores absolutos en relación con la totalidad de la excavación. En algunos casos, se aplicaron distribuciones basadas en la densidad de objetos, es decir, en el número de objetos documentados por retícula de recogida de la información (Hietala et al., 1977; Carbonell et al., 1980; Hietala, 1984).

Actualmente, el uso del teodolito láser permite la toma de puntos tridimensionalmente en relación con un origen externo al yacimiento o en combinación con la posición global terrestre (habitualmente denominado georreferenciación). Esta sistemática permite contextualizar la totalidad del espacio excavado y cada uno de los objetos como parte de un sistema integrado. Este hecho conlleva que la recuperación de estas coordenadas no implique una gran inversión de tiempo.

\section{La excavación en extensión}

Como criterio metodológico, proponemos el de la excavación en extensión, guiada por levantamientos que resiguen la referencia que proporcionan los estratos geológicos. Excavar en extensión no es sinónimo de abrir una superficie de muchos metros cuadrados, error habitual en nuestra profesión, y puede tratarse tanto de pequeñas áreas como de grandes superficies. Excavar en extensión significa trabajar buscando la relación entre los distintos ítems que se van documentando en el registro, ya se trate de objetos o de estructuras. Esta búsqueda está guiada por un proceso hipotético deductivo, en el que las hipótesis se preceden y contrastan con la acumulación de datos que proporciona diariamente la excavación.

Cuando excavamos una unidad arqueológica se va resiguiendo a lo largo de la superficie que hemos planificado excavar. Para ello es necesario identificar el espesor que presenta, por lo que es básico llevar a cabo previamente sondeos que permitan conocer la potencialidad arqueológica del registro que estamos trabajando. Una vez conocida la geometría de la unidad arqueológica, se van efectuando pequeńos levantamientos siguiendo la inclinación y pendiente inicial que proporciona el registro arqueológico recuperado en esos sondeos.

Esta aproximación que vamos realizando paralelamente al proceso de excava- 
ción tiene como objetivo una primera interpretación, que entendemos como básica, ya que está cargada de inputs y observaciones contextuales que se identifican durante el trabajo de campo, pero que desaparecen cuando la interpretación la realizamos en momentos posteriores o es realizada por otros investigadores que no disponen de esas informaciones.

Un primer objetivo buscado con una excavación en extensión es generar un corpus documental que permita la verificación de los datos arqueológicos mediante una contrastación cruzada. Esta estrategia debe partir de una visión integradora de los datos, estableciendo las relaciones existentes entre la entidad real observada (restos óseos, líticos, simbólicos, estructuras, etc.), y las propiedades del objeto deducidas a partir de su estudio (posición estratigráfica, orientación, categoría, etc.). Los atributos dependen del método analítico aplicado y son una cualidad dinámica que puede verse modificada en función del desarrollo de la investigación.

En segundo lugar, se persigue poder llevar a cabo una restitución planimétrica de los datos. Para establecer este entramado de relaciones se precisa la digitalización previa de toda la información de carácter espacial, y en concreto de las estructuras o grandes objetos cuyas dimensiones sean complejas, como son, entre otras cualidades, su posicionamiento simultáneo en más de una unidad estratigráfica, múltiples grados de alteración y rodamiento y relación con la topografía en la que se asientan.

Finalmente, lo que se busca es la interpretación del comportamiento humano y, por ello, es esencial la creación de una base documental con la que argumentar los patrones o modelos a partir de las hipótesis de trabajo contrastadas. Para ello, la información de que disponemos debe ser gestionada por un sistema de registro que reconozca la pluralidad y dinamismo de los datos, que se ajuste a las características del proyecto y que permita el estudio simultáneo de las características espaciales de los objetos donde se han observado dichas variables.

\section{Sistema de registro}

La excavación en extensión requiere que sea cumplimentada con un sistema de recogida de la información que agilice, contextualice $\mathrm{y}$ - a poder ser- universalice los parámetros que hoy consideramos básicos en relación con el objeto arqueológico y su significado. Es por ello que desde 1992 hemos ido desarrollando un sistema de registro que ha sido aplicado a todas las intervenciones arqueológicas que hemos realizado desde el Centre d'Estudis del Patrimoni Arqueològic de la Prehistòria de la Universidad Autónoma de Barcelona (CEPAP-UAB) y que engloba tanto yacimientos al aire libre como en cuevas y abrigos de cronología paleolítica, neolítica y/o paleontológica, contrastando la efectividad de este sistema en un rango cronológico transversal.

El procedimiento seguido en la recogida de la información pasa por cuatro fases principales:

a) adquisición de los datos en soporte magnético; su distribución en bases de datos;

b) digitalización de los soportes con información espacial;

c) integración de todos los datos;

d) generación de mapas temáticos. 
Estos pasos que implementan nueva información al sistema, se mantienen abiertos y en constante modificación permaneciendo operativos cada uno de sus componentes.

\section{Recogida de datos}

Todos los datos espaciales son capturados mediante teodolito láser con distanciómetro óptico incorporado. Este aparato permite almacenar sobre soporte magnético (disco duro, USB, etc.) el resultado de las mediciones y el consiguiente cálculo de las coordenadas cartesianas $(\mathrm{X}, \mathrm{Y}, \mathrm{Z})$ de cada uno de los puntos. De este modo, no es necesario montar una cuadrícula aérea o terrestre física sobre la superficie a excavar, que en algunas ocasiones dificulta y condiciona la visión global y la distribución de los datos. Al igual que no es preciso tener un punto - 0 - , dado que cada punto contiene sus tres dimensiones. Es necesario entender dos elementos básicos para el buen funcionamiento de la dinámica de adquisición de datos y sincronización con el hardware utilizado, que se establecen a partir de los identificadores de los objetos y las estaciones de referencia.

La naturaleza de los datos en una excavación es variada y entre ella podemos mencionar los objetos coordenados, topografías, toma de muestras, forma de las estructuras o de los objetos y georreferenciaciones. Todo ello se nos puede dar en una misma sesión de trabajo. Aquí es donde tiene pleno sentido el identificador, entendido como una cadena alfanumérica junto a un número secuencial, con el que es posible registrar indistintamente datos de procedencia y significado diverso. La cadena alfanumérica, en nuestro caso, consta de ocho dígitos y se utiliza para diferenciar unidades arqueológicas o geológicas y formas. El número secuencial es una cadena numérica que se inicia para cada cadena alfanumérica.

Las estaciones de referencia son indispensables para montar el teodolito láser. Se trata de puntos de ubicación definidos por el usuario y que están dotados de información georreferenciada. Bastará con que conozcamos los parámetros de dos puntos para instalar y montar el teodolito y la consiguiente cuadrícula virtual, a fin de iniciar el trabajo de campo. La ubicación de las estaciones de referencia debe ser pensada detenidamente y buscar los lugares desde los que sea posible visualizar el área en la que se trabaja, para evitar estar cambiando la posición del equipo a lo largo, al menos, de una jornada de trabajo. Las estaciones de referencia van a ser puntos de trabajo que vamos a utilizar continuamente y que en esta primera fase de adquisición de datos van a ser de naturaleza muy diversa.

En los levantamientos topográficos, las superficies ploteadas pueden corresponder al estadio inicial o final en la que se encuentra o se abandona un área de excavación y pueden referirse con superficies o techos de unidades estratigráficas. Igualmente importantes son los perfiles estratigráficos que constituirán una importante fuente de información para posicionar la geometría de las distintas unidades estratigráficas, elemento clave en la contextualización de los objetos arqueológicos, así como con la dinámica de formación y evolución de los estratos.

Es sin embargo dentro del apartado del registro de los materiales arqueológicos donde se contempla una mayor variedad de procedimientos, condicionados esencialmente por el tamaño del 
objeto a coordenar, y si este va a ser levantado en el transcurso de la excavación o, por el contrario, se deja in situ en vistas a una futura musealización, posibilidad reservada solamente a los materiales que se consideren oportunos.

Para todos aquellos objetos de pequeño tamaño o en los que se estima que no es preciso que sean dibujados individualmente, se toma una única coordenada, excepcionalmente dos si queremos documentar la cota superior e inferior del mismo, y se les asigna un «identificador» correspondiente a la unidad arqueológica a la que ha sido adscrito con su respectivo número secuencial. Esta numeración es única y correlativa para todos y a cada uno de los objetos de un mismo nivel, independientemente del área del yacimiento de la que procedan.

La toma de coordenadas tridimensionales permite diferenciar distintas unidades arqueológicas, observar su evolución en los planos horizontal, transversal y sagital de la superficie excavada y posicionar los ítems en sus contextos geológicos, a fin de visualizar la evolución de las estructuras y la dispersión horizontal y vertical de las unidades arqueológicas.

\section{Registro de estructuras arqueológicas}

Cuando el tamaño de los ítems o cualquier otra circunstancia aconsejan que el resto arqueológico sea dibujado, puede ser registrado según dos variantes de un mismo procedimiento, en función de la urgencia con la que deba ser completado el proceso.

En su modalidad más elaborada, el proceso comprende el trazado a mano alzada de un boceto realista del objeto, sin que sea preciso situarlo en relación con ningún otro elemento del entorno y para el que puede escogerse la escala de representación que facilite la labor del dibujante, siempre que esta aporte suficiente detalle. Una vez el boceto está concluido, se toma un número variable de puntos del mismo, identificando estos sobre el dibujo con un número. Es aconsejable que estos puntos se correspondan claramente con partes significativas del dibujo, vértices o fracturas. Una vez en el laboratorio, y con la ayuda del boceto, se reelabora el dibujo del objeto partiendo de la nube de puntos obtenida a partir de las coordenadas tomadas. De vuelta al yacimiento, se verificará el grado de fidelidad en la representación con el elemento original (ya sea un objeto o una estructura) y, en caso de que sea necesario, se procederá a realizar algunas correcciones para mejorar el resultado.

La aplicación de fotografías digitales es un método relativamente simple y con resultados equiparables, ya que parte de un número reducido de puntos fácilmente identificables, de los que se toma la coordenada para su posterior georreferenciación, y puede dibujarse en dos dimensiones. Hoy día es posible generar modelos digitales en tres dimensiones que se pueden georreferenciar a partir de una serie de fotografías.

El registro del objeto, de una u otra forma, sin que se disponga de un equipo informático a pie de campo no puede completarse hasta el día siguiente de haber sido acabado de excavar por completo. Si bien la necesidad de este margen de tiempo puede considerarse una desventaja (y es reducible con el equipamiento adecuado), cuentan como activos la calidad y precisión del resultado final sin que intervenga en ello la experiencia o habilidad del dibujante, puesto que este procedimiento asegura que el resultado 
será independiente de su autor. De un dibujo realizado por este procedimiento puede extraerse información de alta precisión sobre morfometría, orientación, localización, longitud y distancias entre sus fragmentos, etc., al mismo tiempo que permite poner en relación estos o cualquier otro atributo con la morfología de los estratos subyacentes o con otros restos arqueológicos que no sean visibles en el momento de la excavación.

Las estructuras arqueológicas requieren no solo una metodología de excavación específica, sino también la toma de su forma en distintos momentos de su excavación —inicial y final一, así como la delineación que presenta en los planos sagital y transversal a fin de disponer de indicadores sobre su tamaño o volumen y la documentación de los objetos o elementos que la conforman. Es básico documentar las observaciones en cada una de las pautas o fases de trabajo, ya que es muy distinto excavar una estructura de combustión que una fosa. Una vez hayamos finalizado su excavación, tendremos el contexto global de la estructura, aunque en ocasiones estas aparecen delimitadas por secciones estratigráficas o áreas en las que no vamos a excavar inmediatamente, lo que supone que su completa documentación pueda ser transferida a futuros años. El objetivo básico es el de documentar las modificaciones antrópicas que se observan en el espacio en que se sitúan para poder contextualizarlas tanto en la unidad arqueológica como en la secuencia estratigráfica.

\section{La base de datos: La distribución de la información}

La gestión de la información se lleva a cabo diariamente. Todos los datos obte- nidos en una jornada de trabajo de campo son transferidos al ordenador y tratados con el gestor ArqueoUAB, software diseñado para integrar los datos procedentes del entorno tecnológico que se utiliza en el trabajo de campo (teodolito láser y asistentes personales digitales -PDA-).

Programado en Visual Basic 6, permite el almacenamiento y la modificación de datos recuperados en la excavación, así como la realización de consultas diversas, entre las que se encuentra la obtención de secciones de los materiales arqueológicos mediante la generación de ficheros ASCII que incluyen los puntos pertenecientes a una sección definida. A pesar de que ArqueoUAB no es estrictamente un SIG, facilita en gran medida la gestión de la información espacial, algo muy importante en arqueología. Por ejemplo, este sistema ha sido empleado en múltiples yacimientos al aire libre (Mora et al., 2001; Villa et al., 2005) y en abrigos (Martínez-Moreno et al., 2004, Mora et al., 2008).

El cometido principal de este software es facilitar la gestión de la información en cuatro grandes tipos de datos. El identificador con el que hemos tomado cada punto es el que jerarquiza la información y con él quedan discriminados cuatro tipos de contenidos como unidades arqueológicas (UA), datos topográfi$\cos (\mathrm{TO})$, datos geológicos (GE) y morfológicos (FO), y que describimos a continuación.

El grupo UA incluye la información referente a los objetos arqueológicos: unidad arqueológica, coordenadas y una primera clasificación de los materiales según su naturaleza y tipología. Esta clasificación preliminar permite la elabora- 
ción de inventarios de control de los materiales recuperados. Posteriormente, pueden ser adicionados atributos relacionados con la determinación taxonómica de restos óseos o macrovegetales, indicadores tafonómicos, categorías líticas, caracterización de materias primas líticas, registro fotográfico, etc.

En TO se incluyen todos los datos que provienen de las topografías realizadas al inicio y final de la campaña de excavación, así como los de los perfiles estratigráficos. A partir de estos datos, es posible la realización de mapas temáticos en $2 \mathrm{D}$ y en $3 \mathrm{D}$ de distribución en planta de artefactos, pavimentos, o en definitiva, de las superficies recuperadas o generadas en la excavación sobre las que se integran los objetos arqueológicos coordenados y sus dibujos. La correlación espacial de la información procedente de los perfiles estratigráficos permite, a su vez, estudiar la extensión, orientación y geometría de los estratos.

Por su parte, en GE se recoge la posición de todas las muestras que se toman a lo largo de la campańa, sean éstas para análisis palinológicos, carbonatos para análisis isotópicos, etc. Permite disponer de inventarios de todas las muestras tomadas por los distintos especialistas controlando su posición en el espacio, lo que facilita ponerlas en relación con la estratigrafía, la topografía y las unidades arqueológicas.

Por último, en FO se recogen los puntos de referencia de todos los objetos dibujados, bien sean restos óseos, líticos, bloques o estructuras. Contiene múltiples puntos de cada uno de ellos, lo que permite su representación en $2 \mathrm{D}$ y $3 \mathrm{D}$, así como la posibilidad de extraer datos referentes a la orientación y a la pendiente real de los objetos.

\section{Digitalización de soportes}

Los restos arqueológicos dibujados o fotografiados en la excavación son vectorizados aplicando programas SIG. Para una gestión más ágil del archivo resultante, cada ítem, ya sea un hueso de grandes dimensiones o un bloque desprendido, se almacena en una capa independiente, cuyo nombre es una cadena codificada que incluye información del nivel estratigráfico, número de identificación de la pieza y principales características. Este campo se halla también de forma idéntica en la base de datos, lo que permite relacionar estas tablas entre sí con la entidad espacial representada por el objeto dibujado.

La digitalización de los restos arqueológicos es un proceso laborioso, aunque se ve compensado por ser un trabajo que, una vez realizado, se constituye en un soporte espacial relacionable con cualquiera de sus atributos. Con las actuales técnicas de fotogrametría combinadas con las capacidades de geoposicionamiento de la base de datos, es posible la georreferenciación de fotografías de la excavación. Esta herramienta es de especial interés para la restitución de estructuras de combustión, así como para la confección de estratigrafías de los sondeos y la recreación de cualquier tipo de forma. Además, es posible la creación de modelos 3D georreferenciados que se integran con el resto de datos recuperados en el yacimiento de forma virtual.

\section{Gestión integrada y mapeado: el uso del GIS}

Para la gestión de los datos se ha escogido ArcGIS, software SIG (Sistemas de Información Geográfica) muy extendido 
en el mercado. La información está organizada y dispuesta de tal manera que su gestión es casi automática. Las capacidades de este tipo de explotación son muy amplias y actualmente constituyen vías de desarrollo muy prometedoras para la arqueología (Llobera, 2003; Owens et al., 2014).

Una vez los datos de excavación han sido transferidos a la base de datos, la información georreferenciada es accesible mediante un sistema de coordenadas cartesianas en el cual las variables X, Y, Z se expresan en milímetros. La proyección de las coordenada X e Y permite generar planimetrías (plantas) de las entidades excavadas. Por otra parte, con la combinación de la variable $\mathrm{Z}$, con las coordenadas $\mathrm{X}$ o $\mathrm{Y}$ se obtienen perfiles transversales, sagitales y oblicuos que permiten visualizar la geometría de las unidades arqueológicas.

El trabajar con una base de datos relacional permite la integración de la información cualitativa y cuantitativa que define al objeto arqueológico y contribuye a la interpretación del contexto arqueológico (Martínez-Moreno et al., 2004). Esta aproximación asume el principio de que los objetos arqueológicos se comportan como partículas sedimentarias, que tienen un complejo proceso tafonómico en su tránsito desde la biosfera hasta la litosfera (Gifford, 1981). Consecuentemente, es necesario analizar la naturaleza de las relaciones entre los artefactos y los procesos que han sufrido, a fin de determinar cómo están conectados y los procesos a que han sido sometidos a lo largo del tiempo.

\section{Conclusión}

El objeto arqueológico es algo más que los atributos que lo conforman, ya que se integra dentro de un contexto, a veces no evidente, que lo redimensiona y define. Este contexto se va configurando conforme avanza el proceso de excavación, siendo ese carácter dinámico una de sus principales características.

Puesto que una excavación es un sistema abierto en el que las observaciones del investigador son importantes en su interpretación, proponemos acotar la subjetividad del proceso a través de una metodología basada en la excavación en extensión y un sistema de registro exhaustivo y minucioso.

El sistema de registro es una herramienta esencial a la hora no solo de emitir inferencias sobre la organización de los grupos humanos del pasado, sino también para evaluar la validez de las inferencias que desde el presente nosotros emitimos. Como hemos tratado de exponer a lo largo de esta presentación, generar un registro arqueográfico preciso es absolutamente necesario para avanzar en cualquier investigación.

La mayoría de las observaciones que se realizan en campo durante la recuperación del registro arqueológico a veces no quedan registradas, ya sea porque pasan inadvertidas o simplemente porque durante la excavación no somos capaces de verificarlas. La contextualización de un objeto arqueológico no es única, sino que, conforme se avanza en la excavación, se van añadiendo rasgos, elementos o atributos que no se pudieron identificar en el momento en que fue recuperado. La restitución del objeto en su contexto arqueológico y estratigráfico, mediante la realización de plantas y 
secciones, se convierte en una herramienta básica con la que poder generar hipótesis de trabajo. Estas, a su vez, deberán ser testadas a medida que se obtengan y actualicen con nuevos datos.

Somos conscientes de que las técnicas que proponemos no conforman una innovación; al contrario, se trata de técnicas simples y ampliamente empleadas en la arqueología de campo. Sin embargo, consideramos que es relevante incidir en la dinámica que se deriva de la aplicación de este enfoque, que supera esa perspectiva estática con la que habitualmente nos aproximamos a la interpretación del registro arqueológico.

Por otro lado, el uso de aparatos topográficos e infraestructuras digitales, así como su posterior gestión por medio de sistemas microinformáticos, suponen una gran ayuda durante la realización de los trabajos de campo, al disponer de elementos esenciales, como la distribución en la superficie y la dispersión vertical de una unidad arqueológica. Su delineación, densidad, las estructuras que la conforman, la presencia o ausencia de determinadas categorías o materiales, son elementos que pueden articular y guiar la organización de los trabajos de campo.

Es decir, esta sistemática de trabajo se convierte en una herramienta con la que afrontar los múltiples y habituales retos que plantea la excavación de un yacimiento, máxime en el tipo de yacimientos que hemos tratado en este artículo difíciles de visualizar y en los que es raro detectar elementos que permitan una rápida identificación a nivel espacial.
El uso de esta metodología no aporta una solución definitiva a esta cuestión. Sin embargo, permite diseñar hipótesis que pueden ser contrastadas, es decir, que validen o refuten esas observaciones. A partir de este conocimiento provisional, que construye a medida que se va excavando, pueden adoptarse respuestas que o bien permitan continuar con esa sistemática o, alternativamente, reorientar completamente la dirección de esas decisiones.

La flexibilidad de este método es una ventaja que consideramos que hace a esta propuesta relevante para comprender y explicar un aspecto siempre complejo como es el de intentar hacer hablar a un registro fósil, que habitualmente no es fácil de interpretar.

Este método, que deriva de los trabajos iniciales de Meroc y Laplace (1954) y que afecta a la totalidad del registro arqueológico (restos óseos o líticos), a los levantamientos macro o microestratigráficos o de cualquier otro tipo que conforman un yacimiento, es implementado con una gestión relacionada con la aplicación de sistemas de información geográfica. Actualmente el software y el hardware necesarios para su aplicación son de uso común, y tan solo se requieren algunos desarrollos de software propios para agilizar algunos de los pasos más tediosos.

Independientemente del método o la técnica que se use, es necesario un planteamiento metodológico: excavación en extensión, dirigida a interrelacionar los distintos ítems o estructuras y crear hipótesis de trabajo que hemos de contrastar. 


\section{Agradecimientos}

Las investigaciones del Centre d'Estudis del Patrimoni Arqueològic de la Prehistòria (CEPAP-UAB) forman parte del proyecto «Poblamiento humano durante el pleistoceno final e inicios del holoceno en el sureste de los Pirineos (HAR2010-15002, HAR2013-42338)» y del grupo de investigación 2014SGR-0084. Miquel Roy Sunyer disfruta de una beca FI de la Generalitat de Catalunya. Xavier Roda Gilabert es beneficiario de la Ayuda FPI-MINECO (BES-2011-045180).

\section{Referencias bibliográficas}

Carbonell, E.; Mora, R.; Canal, J. 1980. Sota Palou: un campament estacional-climàtic de caçadors prehistòrics. IV Col.loqui internacional d'arqueologia de Puigcerdà. Estat actual de la recerca arqueològica a l'istme pirinenc. Puigcerdà: Institut d'Estudis Ceretans.

Dibble, H. 1987: «Measurement of artefact provenience with an electronic theodolite». Journal of Field Archaeology 14, 249-54.

Dibble, H. L.; McPherron, S. 1998. "On the computerization of archaeological projects». Journal of Field Archaeology 15, 431-40. $<$ http://dx.doi.org/10.1179/jfa.1988.15.4.431>

Dibble, H.; Marean, C.; McPherron, S. 2007. "On the use of barcodes in excavation projects with examples from Mossel Bay (South Africa) and Roc de Marsal (France)». The SAA Archaeological Record 7(1), 33-38.

Dibble, H.; Mcpherron, S.; Sandgathe, D.; Goldberg, P.; Turo, A.; Lenoir, M. 2009. "Context, curation, and bias: an evaluation of the Middle Paleolithic collections of CombeGrenal (France)». Journal of Archaeological Science 36, 2540-50. $<$ http://dx.doi.org/10.1016/j.jas.2009.07.010>

Dibble, H.; Raczer, T.; Mcpherron, S. 2005. «Excavator Bias at the Site of Pech de l'Azé IV (France)». Journal of Field Archaeology 30, 317-28. <http://dx.doi.org/10.1179/009346905791072242>

Gifford, D. 1981. «Taphonomy and Palaeocology: a critical review of archaeology's sister discipline». En: Schiffer (ed.). Advances in Archaeological Method and Theory 4, 77-101. Academic Press.

Hietala, H. J.; Stevens, D. E. 1977. «Spatial analysis: multiple procedures in pattern recognition studies». American Antiquity 42(4): 539-59. $<$ http://dx.doi.org/10.2307/278927>

Hietala, H. J. 1984. Intrasite spatial analysis in archaelogy. Cambridge: Cambridge University Press.

Laplace, G. 1971. «De l'application des coordonnés à la fouille stratigraphique». Munibe (Antropologia y Arqueologia) 2-3: 19-71.

Llobera, M. 2003 «Extending GIS-based visual analysis: the concept of visualscapes». International Journal of Geographical Information Science 17, 25-48.

Martínez-Moreno, J.; Mora, R.; De la Torre, I. 2004. «Methodological approach for understanding Middle Palaeolithic settlement dynamics at La Roca dels Bous (Noguera, 
Catalunya, Northeast Spain)». En: Conard (ed). Settlement Dynamics of the Middle Palaeolithic and Middle Stone Age II, 393-413.

Martínez-Moreno, J.; Mora, R.; De la Torre, I. En prensa. The Middle-To-Upper Palaeolithic.

Mcpherron, S. 2005: «Artifact orientations and site formation processes from total station proveniences». Journal of Archaeological Science 32, 1003-14. $<$ http://dx.doi.org/10.1016/j.jas.2005.01.015>

Mcpherron, S.; Dibble, H. 2003. «Using computers in adverse field conditions». The SAA Archaeological Record 3 (5), 28-32.

Méroc, L.; Laplace-Jauretche, G. 1954. «Application des coordonnées cartésiennes à la fouille d'un gisement». Bulletin de la Société préhistorique de France: 58-66.

Mora, R.; Martínez-Moreno, J.; Casanova, J. 2008. "Abordando la noción de "variabilidad musteriense" en Roca dels Bous». Trabajos de Prehistoria 65, 13-28.

Mora, R.; Parcerisas, J; Martínez, J. 2001. "Computer-based recording systems of Pleistocene deposits with large mammals». En: The World of Elephants/La Terra degli Elefanti. Proceedings of the First International Congress, 219-24. Roma.

Owens, J. B.; Sandes, A.; Stephenson, B.; Dixon, D.; Zajanc, C. 2014. "A Geographic Information Systems (GIS) Training Manual for Historians and Historical Social Scientists». Recurso electrónico: <http://www.geographicallyintegratedhistory.com> «Transition in Cova Gran (Catalonia, Spain) and the extinction of Neanderthals in the Iberian Peninsula». Journal of Human Evolution.

Villa, P.; Courtin, J. 1983. «The interpretation of stratified sites. A view from underground». Journal of Archaeological Science 10, 267-81. <http://dx.doi.org/10.1016/0305-4403(83)90011-0>

Villa, P.; Soto, E.; Santonja, M.; Pérez-González, A.; Mora, R.; Parcerisas, J.; Sesé, C. 2005. «New data from Ambrona: closing the hunting versus scavenging debate». Quaternary International 126-128, 223-50.

<http://dx.doi.org/10.1016/j.quaint.2004.03.001> 